\title{
Effects of osteopontin-enriched formula on lymphocyte subsets in the first 6 months of life: a randomized controlled trial
}

\author{
Christina E. West ${ }^{1}$, Anne Staudt Kvistgaard ${ }^{2}$, Janet M. Peerson ${ }^{3}$, Sharon M. Donovan ${ }^{4}$, Yong-mei Peng ${ }^{5}$ and Bo Lönnerdal ${ }^{3}$
}

BACKGROUND: Human milk is rich in osteopontin (OPN), which has immunomodulatory functions.

METHODS: In a randomized controlled trial, standard formula (SF) and the same formula with $65 \mathrm{mg}$ of OPN/L (F65) or 130 mg of OPN/L (F130), representing $~ 50$ and 100\% of the OPN concentration in human milk, were compared. We examined frequencies and composition of peripheral blood immune cells by four-color immunoflow cytometry of formula-fed infants at ages 1, 4, and 6 months, and compared them with a breastfed (BF) reference group.

RESULTS: The F130 group had increased T-cell proportions compared with the SF ( $P=0.036$, average effect size 0.51$)$ and F65 groups ( $P=0.008$, average effect size 0.65). Compared with the BF group, the monocyte proportions were increased in the F65 ( $P=0.001$, average effect size 0.59$)$ and F130 $(P=0.006$, average effect size 0.50) groups, but were comparable among the formula groups.

CONCLUSION: OPN in an infant formula at a concentration close to that of human milk increased the proportion of circulating $T$ cells compared with both SF and formula with added OPN at $\sim 50 \%$ of the concentration in human milk. This suggests that OPN may favorably influence immune ontogeny in infancy and that the effects appear to be dose-dependent.

$\mathbf{T}$ he newborn infant is particularly susceptible to infection, and there is clear evidence that breastfeeding is associated with reduced number of infections in infancy, particularly in developing countries; however, breastfeeding may also confer protection against otitis media and gastrointestinal infections in developed countries (1). This is highly likely to be mediated by the large variety of bioactive components present in human milk (2). Human milk components are also implicated in the maturation of innate and adaptive immunity $(3,4)$ in commensal gut microbiota establishment (5) and in protection against inflammation (6). In addition to cytokines, chemokines, and growth factors, many of these bioactive components are peptides and proteins, and there is emerging interest in the role of milk-borne osteopontin (OPN) in pediatric nutrition (7). OPN is a multifunctional protein involved in physiological processes such as wound healing, angiogenesis, biomineralization, tissue remodeling, and also in immune activation, including T helper 1 (Th1) polarization and recruitment of monocytes and macrophages $(2,8)$. Although originally identified in bone, OPN was later reported to be present in human milk at a high concentration, i.e., $\sim 130 \mathrm{mg} / \mathrm{l}$, and at a much lower concentration in bovine milk, and therefore also at lower concentrations in infant formula (2). Formulas are usually based on whey protein concentrate and skim milk, which are mixed in certain proportions, but as bovine milk is low in OPN it will be low in both these materials.

OPN is expressed in various tissues and cell types, it induces signal transduction pathways via integrins and CD44 variants, and it is a potent chemoattractant for many cell types including macrophages, dendritic cells, and T cells $(8,9)$. A synonymous name for OPN is Eta-1 (early $\mathrm{T}$ lymphocyte activation gene-1) because of its high expression in activated $\mathrm{T}$ cells $(8,9)$. OPN is strongly upregulated in response to T-cell receptor (TCR) ligation (10), and mediates migration and adhesion, as well as co-stimulates T-cell proliferation (9). OPN is considered a key molecule inducing Th-1-type immunity; OPN gene expression in $\mathrm{T}$ cells is controlled by $\mathrm{T}$-cell transcription factor regulating $\mathrm{T}$ helper 1 cell lineage commitment, $\mathrm{T}$ bet, and $\mathrm{T}$ bet-dependent $\mathrm{OPN}$ expression in $\mathrm{T}$ cells is also critical for skewing of CD4+ T and CD8+ T cells in the direction of the Th1 and type 1 CD8 T-cell (Tc1) pathways (10). This is of potential interest in early infancy, as term newborns in the first months of life not only have impaired adaptive immune responses but also have low innate Th1 support and low innate antiviral interferon production (11). Studies in OPN-null mice have shown that OPN enhances host defense against many microorganisms including HSV1 and rotavirus $(12,13)$. A potential role for OPN in mediating host defense is further corroborated by the observation that Mycobacterium bovus bacillus CalmetteGuerin (BCG) grew more rapidly in macrophages derived from OPN-null mice (14). Additionally, tissue OPN expression and disease progression correlated inversely following

\footnotetext{
${ }^{1}$ Department of Clincial Sciences, Pediatrics, Umeå University, Umeå, Sweden; ${ }^{2}$ Arla Foods Ingredients, Viby, Denmark; ${ }^{3}$ Department of Nutrition, University of California, Davis, California; ${ }^{4}$ Department of Food Science and Human Nutrition, University of Illinois, Urbana, Illinois; ${ }^{5}$ Department of Pediatrics, Fudan University, Shanghai, China. Correspondence: Bo Lönnerdal (bllonnerdal@ucdavis.edu)

Received 18 June 2016; accepted 19 February 2017; advance online publication 24 May 2017. doi: 10.1038/pr.2017.77
} 


\section{Articles | west et al.}

inoculation with BCG in human subjects (15). In infants, breastfeeding was reported to increase proliferative responses of $\mathrm{T}$ cells following vaccination with BCG at birth compared with formula-feeding (16), which could be at least partly an effect of milk-borne OPN.

We hypothesized that the addition of OPN in infant formula would impact immune cell composition in the very young infant. Our aim was to evaluate whether bovine OPN added to infant formula at a concentration of $\sim 50$ or $100 \%$ of that of human milk would affect immune cell composition in blood in the nearly newborn and in the first half of infancy. As there is only one small prospective study that has followed immune cell ontogeny in formula-fed (FF) compared with breastfed (BF) infants (17) we also aimed at evaluating immune cell development in the first 6 months of life in infants fed standard formula or experimental formula with bovine OPN, in comparison with a BF reference group.

\section{RESULTS}

\section{Study Subjects}

From the initiation of the intervention (one month of age), the dropout rates were $2 \%, 10 \%, 11 \%$, and $23 \%$ in the SF, F65, F130, and BF groups, respectively (Figure 1) (18). There was no significant difference in dropout rate among the formula groups $(P=0.096$, Fisher's exact test). The most common reason for dropping out of the BF group was the perception of insufficient milk production and inadequate phlebotomy in the formula groups. In the present study, we included all infants with successful venipunctures at the ages 1, 4, and 6 months. The number of infants included in the analysis of peripheral blood leukocyte composition are displayed in Table 2. For phenotyping by immunoflow cytometry, a relatively large blood volume was needed and an adequate sample volume for analysis was obtained from 37, 34, and 34 infants in the BF group; 33, 31, and 33 infants in the SF group; 30,29 , and 26 infants in the F65 group; and 37, 33, and 34 infants in the F130 group, at 1, 4, and 6 months of age, respectively (Figure 1).

\section{WBC and Leukocyte Subsets in the Formula Groups and in the} BF Reference Group

Repeated measures analysis revealed no differences in the total WBC count between the formula groups or when comparing the formula groups with the $\mathrm{BF}$ reference group (Table 2). Lymphocyte counts increased from 1 to 6 months of age, and there was an interaction of type of feeding and time $(P=0.026)$. The average effect size from 1 to 6 months of age was 0.22 in the combined formula group and 0.49 in the $\mathrm{BF}$ reference group, indicating a small to moderate effect. Follow-up analyses showed no differences in lymphocyte counts among the formula groups, or between the formula and the BF group (Table 2). There were no differences among the formula groups in monocyte counts, but the BF group had lower monocyte counts than the combined formula group (Table 2) $(P<0.0001)$. The average effect size was 0.80 , indicating a moderate to large effect. Eosinophil counts increased from 1 to 6 months of age and there was an interaction of type of feeding and time $(P=0.01)$. The average effect size from 1 to 6 months of age was 0.08 in the combined formula group and 0.13 in the BF reference group, indicating a small effect. Follow-up analyses revealed no differences in eosinophil counts between the formula groups or when comparing the combined formula group with the BF reference group (Table 2). Basophil and neutrophil counts remained unchanged from 1 to 6 months of age, with no differences among the formula groups or when comparing the combined formula groups with the BF reference group (Table 2).

\section{The Proportion of T Cells was Higher in the F130 Group Compared with the Other Formula Groups}

To distinguish lymphocyte subtypes, we used a panel of monoclonal antibodies in four-color immunoflow cytometry (Figure 2). Generally, T cells, CD3+/CD45+, represented the dominant lymphocyte population at all blood samplings (Figure 2a). Even though infants in the F130 group had the highest proportion of $\mathrm{T}$ cells already at entry, repeated measures analyses revealed that the increased proportion of $\mathrm{T}$ cells throughout the intervention in the F130 group was statistically significantly different compared with both the SF $(P=0.036)$ and $\mathrm{F} 65$ groups $(P=0.008)$ but not in comparison with the $\mathrm{BF}$ reference group $(P=0.093$; Figure 2b). The average effect size when comparing the F130 group with the SF group was 0.51 , and the corresponding value for the F130 group versus the F65 group was 0.65 , indicating a moderate effect. There was no difference between the formula groups in the proportion of $\mathrm{T}$ helper cells, CD3+CD4 $+/ \mathrm{CD} 45$ (Figure 2c), nor in the $\mathrm{CD} 4 / \mathrm{CD} 8$ ratio $(P>0.05$; data not shown). Infants in the F130 group displayed the highest proportion of cytotoxic T cells, CD3+CD8+/CD45, although this difference was not statistically significant in comparison with the other formula groups or with the $\mathrm{BF}$ reference group (Figure $2 \mathbf{d}$ ).

\section{Differences in Immune Cell Composition Between the Combined Formula Groups and the BF Reference Group} We also examined the proportions of circulating monocytes, characterized as CD45+CD14+ cells. Even though there were no statistically significant differences among the formula groups, repeated measures analyses revealed that the proportion of circulating monocytes was augmented in the two groups randomized to experimental formula in comparison with the BF group. Monocyte proportions were increased in the F65 $(P=0.001$, average effect size 0.59$)$ and F130 $(P=0.006$, average effect size 0.50$)$ groups, compared with the $\mathrm{BF}$ group, indicating a moderate effect. In contrast, the proportion of circulating monocytes in infants randomized to standard formula was comparable to that of the BF reference group (Figure 3a).

Some additional differences between $\mathrm{BF}$ and $\mathrm{FF}$ infants also emerged. First, the proportion of naive T cells, CD45RA+CD3+, was higher in $\mathrm{BF}$ infants compared with $\mathrm{FF}$ infants $(P=0.03)$ in the first 6 months of life (Figure 3b), although 


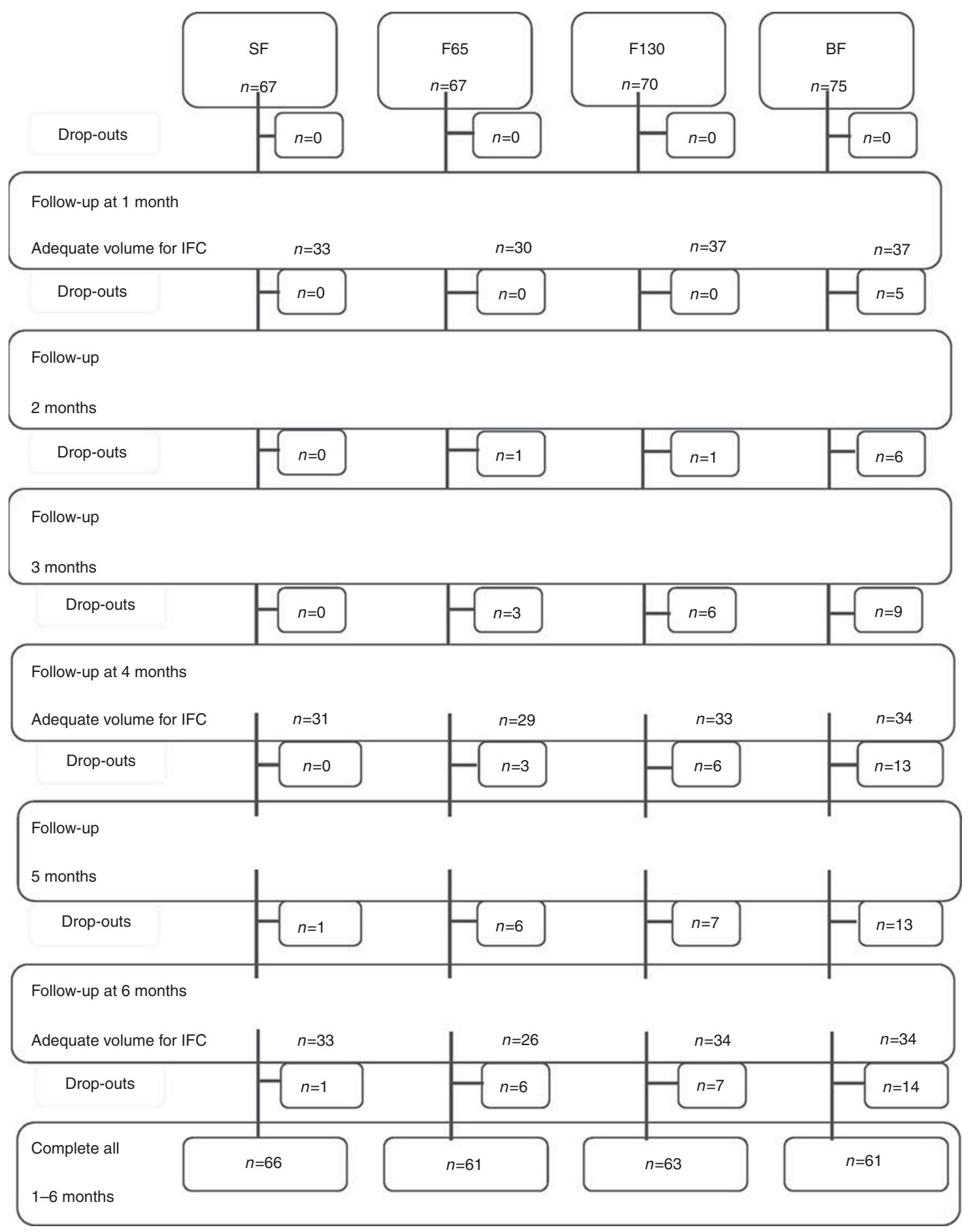

Figure 1. Trial profile. Number of subjects in each group at the start and each month of the intervention, as well as dropouts and subjects with adequate blood volume for immunoflow cytometry (IFC).

an average effect size of 0.13 indicates a small effect. However, already at one month of age, the mean (SD) proportion (\%) of circulating memory and/or activated $\mathrm{T}$ cells, $\mathrm{CD} 45 \mathrm{R} 0+\mathrm{CD} 3+$, was 15.2 (5.4) in BF infants and 12.9 (3.7), 13.7 (5.1), and 13.8 (4.9) in the SF, F65, and F130 groups, respectively. No differences in the proportions of this subset according to type of feeding emerged (Figure 3c). As both activated $\mathrm{T}$ cells and memory $\mathrm{T}$ cells express CD45R0, we also examined the proportion of the active phenotype that expresses the MHC class II molecule HLA-DR-i.e., HLA-DR+CD3+ cells. This subset varied between 10 and $15 \%$ at all samplings, with no differences according to type of feeding (Figure 3d). A small 


\section{Articles | West et al.}

Table 1. Nutrient composition of the standard infant formula (SF)

\begin{tabular}{|c|c|c|c|}
\hline \multirow[b]{2}{*}{ Composition } & \multicolumn{2}{|r|}{ Target value } & \multirow[b]{2}{*}{$\begin{array}{l}\text { per } 100 \\
\text { kcal }\end{array}$} \\
\hline & Units & $\begin{array}{l}\text { per } 100 \mathrm{~g} \\
\text { powder }\end{array}$ & \\
\hline Energy & $\begin{array}{l}\mathrm{kcal} / \\
\mathrm{kJ}\end{array}$ & $500 / 2093$ & \\
\hline Protein & $g$ & 11.00 & 2.20 \\
\hline Whey proteins & $g$ & 7.7 & 1.5 \\
\hline Casein & $g$ & 3.3 & 0.7 \\
\hline a-lactalbumin & g & 1.9 & 0.4 \\
\hline Fat & $g$ & 25.0 & 5.0 \\
\hline Linoleic acid & $\mathrm{mg}$ & 3700 & 740.3 \\
\hline a-linolenic acid & $\mathrm{mg}$ & 450 & 90.0 \\
\hline Linoleic acid/a-linolenic acid & & 8.22 & 8.22 \\
\hline DHA & $\mathrm{mg}$ & 50 & 10.0 \\
\hline ARA & $\mathrm{mg}$ & 80 & 16.0 \\
\hline$\% \mathrm{DHA} /$ total fatty acids & & 0.21 & 0.21 \\
\hline$\%$ ARA/total fatty acids & & 0.33 & 0.33 \\
\hline $\begin{array}{l}\text { 1,3-Dioleoyl 2-palmitoyl } \\
\text { triglyceride }\end{array}$ & $g$ & 4.75 & \\
\hline Carbohydrate & $g$ & 56.7 & 11.3 \\
\hline GOS & $\mathrm{g}$ & 2.0 & 0.4 \\
\hline Nucleotides & $\mathrm{mg}$ & 22.0 & 4.4 \\
\hline Vit. A & $\mu \mathrm{g} R$ & 550 & 110 \\
\hline Vit. D & $\mu \mathrm{g}$ & 10.6 & 2.1 \\
\hline Vit. $E$ (a-TE) & $\mathrm{mg}$ & 7.0 & 1.4 \\
\hline Vit. K & $\mu \mathrm{g}$ & 45.0 & 9.0 \\
\hline Vit. B1 & $\mu \mathrm{g}$ & 900.0 & 180.1 \\
\hline Vit. B2 & $\mu \mathrm{g}$ & 1002.0 & 200.5 \\
\hline Vit. B6 & $\mu \mathrm{g}$ & 600.0 & 120.0 \\
\hline Vit. B12 & $\mu \mathrm{g}$ & 1.65 & 0.3 \\
\hline Vit. C & $\mathrm{mg}$ & 100 & 20.0 \\
\hline L-carnitine & $\mathrm{mg}$ & 9.0 & 1.8 \\
\hline Niacin & $\mu \mathrm{g}$ & 4100.0 & 820.3 \\
\hline Pantothenic acid & $\mu \mathrm{g}$ & 3500 & 700 \\
\hline Folic acid & $\mu \mathrm{g}$ & 120 & 24.0 \\
\hline Biotin & $\mu \mathrm{g}$ & 20 & 4.0 \\
\hline Taurine & $\mathrm{mg}$ & 40 & 8.0 \\
\hline Choline & $\mathrm{mg}$ & 100 & 20.0 \\
\hline Inositol & $\mathrm{mg}$ & 40 & 8.0 \\
\hline Calcium & $\mathrm{mg}$ & 351 & 70.2 \\
\hline Potassium & $\mathrm{mg}$ & 686 & 137.3 \\
\hline Phosphorus & $\mathrm{mg}$ & 200 & 40.0 \\
\hline Calcium/phosphorus & & 1.76 & 1.76 \\
\hline Iron & $\mathrm{mg}$ & 5.0 & 1.0 \\
\hline Zinc & $\mathrm{mg}$ & 3.8 & 0.8 \\
\hline
\end{tabular}

Table 1 Continued

\begin{tabular}{|c|c|c|c|}
\hline \multirow[b]{2}{*}{ Composition } & \multicolumn{2}{|r|}{ Target value } & \multirow[b]{2}{*}{$\begin{array}{l}\text { per } 100 \\
\text { kcal }\end{array}$} \\
\hline & Units & $\begin{array}{l}\text { per } 100 \mathrm{~g} \\
\text { powder }\end{array}$ & \\
\hline Copper & $\mu \mathrm{g}$ & 400.0 & 80.0 \\
\hline lodine & $\mu g$ & 120.0 & 24.0 \\
\hline Sodium & $\mathrm{mg}$ & 150.0 & 30.0 \\
\hline Chloride & $\mathrm{mg}$ & 331.0 & 66.2 \\
\hline Manganese & $\mu \mathrm{g}$ & 176.0 & 35.2 \\
\hline Selenium & $\mu \mathrm{g}$ & 15 & 3.0 \\
\hline
\end{tabular}

proportion of $\mathrm{T}$ cells was $\mathrm{CD} 45 \mathrm{R} 0+\mathrm{RA}+\mathrm{CD} 3+$, with no differences according to type of feeding (data not shown).

Next, we examined the proportion of double-positive $\mathrm{T}$ cells, CD3+CD4+CD8+. The proportion of this subset of circulating immature $\mathrm{T}$ cells was low, $<2 \%$ in all groups, but it was higher in the combined formula group during the intervention compared with the $\mathrm{BF}$ group $(P<0.001)$. The average effect size was 0.12 , indicating a small effect. No differences between the formula groups emerged (Figure 3e). $\mathrm{T}$ cells were then differentiated according to TCR usage. As expected, the majority of T cells expressed TCR- $\alpha \beta$ irrespective of the type of feeding (Figure 3f). The proportion of $\alpha \beta$ $\mathrm{T}$ cells was higher in the BF reference group compared with the combined formula group $(P<0.0001)$, with an average effect size of 0.67 . In contrast, the proportion of TCR- $\gamma \delta+$ cells was higher in the combined formula group compared with the BF reference group $(P=0.0003$; Figure $3 g)$, with an average effect size of 0.60 . A small proportion $(<1 \%)$ of cells was TCR- $\alpha \beta+\gamma \delta+$, and this proportion was higher in the combined formula group compared with the $\mathrm{BF}$ reference group (data not shown; $P<0.001$ ), with an average effect size of 0.76 .

$\mathrm{B}$ cells constituted the second largest population and increased as expected in proportion in both $\mathrm{BF}$ and $\mathrm{FF}$ infants $(P$ for time $=0.003$ ), with an effect size of 0.84 from 1 to 6 months of age and with no differences between BF and FF infants (Figure $3 \mathbf{h}$ ). The proportion of NK cells, CD16 $+\mathrm{CD} 56+$, remained unchanged from 1 to 6 months of age, irrespective of the type of feeding (Figure 3i).

\section{DISCUSSION}

This is the first study to prospectively examine the effects of OPN added to infant formula on immune cell composition in very young infants. Our main findings are that an experimental formula with an OPN concentration close to that of human milk increased the proportion of $\mathrm{T}$ cells in the first 6 months of life compared with standard formula. Further, the proportion of circulating monocytes was higher in the two groups receving experimental formulas with added OPN at a concentration of 50 and $100 \%$ of that of human milk, 
compared with a BF reference group. Additional differences between $\mathrm{BF}$ and $\mathrm{FF}$ infants were also seen; the proportion of naive $\mathrm{T}$ cells, CD45RA+CD3+, was slightly higher in the $\mathrm{BF}$ reference group compared with the combined formula group during the first 6 months of life, although the proportion of the memory/and or activated pheontype, CD45R0+CD3+, was similar. Compared with BF infants, the proportions of double-positive CD4+CD8+ cells, $\gamma \delta \mathrm{T}$ cells, and $\alpha \beta \gamma \delta \mathrm{T}$ cells were higher in the combined formula group, whereas the proportion of $\alpha \beta T$ cells was lower. Collectively, this underscores that immune cell composition and ontogeny are influenced by breastfeeding, and that formula-feeding with added bioactive OPN may increase the proportions of T cells and monocytes, but that the effect appears to be dosedependent.

Higher concentrations of interleukin 2 (IL-2), which is central for T-cell growth and differentiation (19), were also observed in the groups that received experimental formula with added OPN in comparison with both the SF and BF groups (18). In experimental granuloma models, OPN has been shown to bind to $\mathrm{T}$ cells, to support $\mathrm{T}$-cell adhesion and chemotaxis, and also to co-stimulate resting $\mathrm{T}$-cell proliferation (20). Increased proportions of $\mathrm{T}$ cells in FF compared with BF infants at 6 months of age (21) and between 4 and 6 months of age (17) have previously been reported using other types of formula. In disease states, increased $\mathrm{T}$-cell proportions and persistent T-cell activation can be harmful, but in our setting the proportions of $\mathrm{T}$ cells followed the previously reported pattern with an age-dependent decrease $(22,23)$ in the first half of infancy in both BF and FF infants irrespective of OPN content, and with no differences in the proportion of the active phenotype, i.e., HLA-DR+CD3+ cells, between the BF and FF groups. Second, the proportion of monocytes was higher in both groups receving experimental formula with added OPN at a concentration of $50 \%$ or $100 \%$ of that of human milk, compared with the BF reference group. OPN is a potent chemoattractant $(8,9)$ for many cell types including monocytes and T cells. In early infancy, monocyte function is immature and proinflammatory innate immune responses increase over the first months of life (24). An increase in the circulating monocyte proportion could be of importance and warrants further study to determine whether formula with added OPN may also enhance the functional capacity in innate immunity in neonates.

A number of differences between $\mathrm{BF}$ and $\mathrm{FF}$ infants were also seen. The proportion of naive CD45RA+CD3+ $\mathrm{T}$ cells was slightly higher in BF compared with FF infants in the first half of infancy, whereas the mean proportion of circulating memory and/or activated $\mathrm{T}$ cells, CD45R0+CD3+, varied between 12 and $16 \%$ in $\mathrm{BF}$ and $\mathrm{FF}$ infants, with no differences according to feeding mode. As the central site of T-cell development, there has been much interest in the effects of early feeding on thymus size and function. Thymus is highly active in the neonatal period, and BF infants have a larger thymus than FF infants $(25,26)$. Even in adults, thymic tissue was positively associated with circulating naive T cells in HIV 


\section{Articles | West et al.}
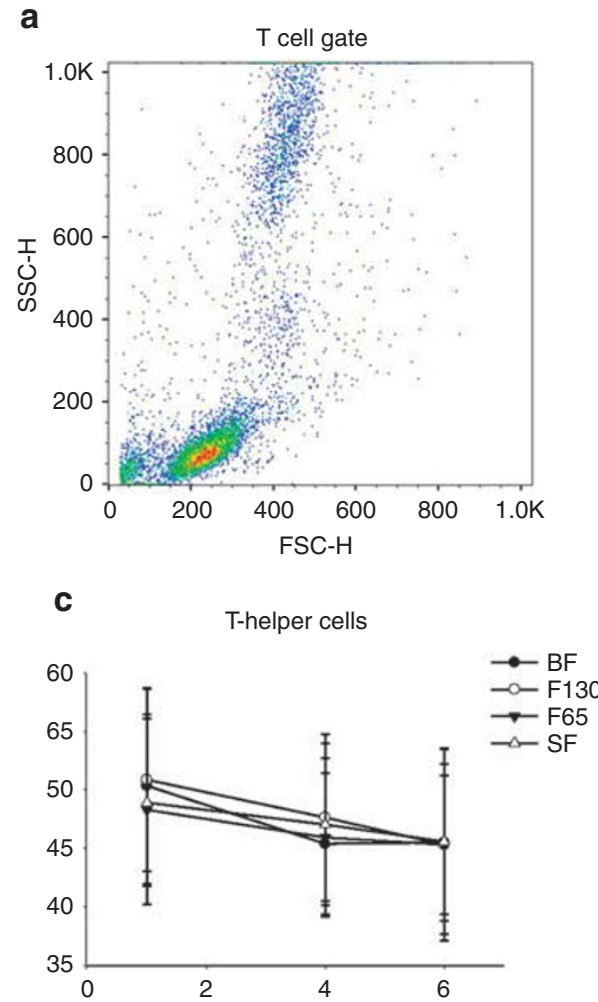
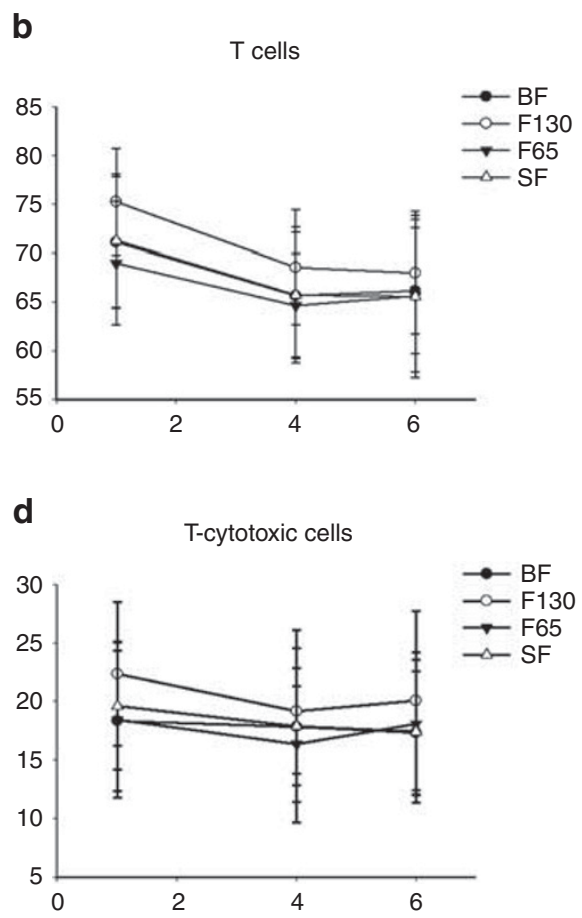

Figure 2. Immune cell composition was analyzed with four-color immunoflow cytometry of PBMCs of infants in the BF reference group and the three formula groups. (a) Representative flow cytometry plot of a 1-month-old infant showing the T-cell gate. (b) Mean proportions of T cells (CD3 $+/ C D 45+)$. (c) T helper cells (CD3+CD4+/CD45). (d) Cytotoxic T cells (CD3+CD8+/CD45). Bars represent mean (SD). The mean proportion of T cells was increased in the F130 group compared with both the SF $(P=0.036$, average effect size 0.51$)$ and F65 groups $(P=0.008$, average effect size 0.65$)$.

infection (27). It is well recognized that IL-7 is important for T-cell development in the thymus (28) and thymic function in exclusively BF infants is positively associated with IL-7 concentrations in human milk (29). Recently it was shown that milk-derived IL-7 can cross the intestinal barrier and augment subsets of thymocytes and peripheral $\mathrm{T}$ cells in mice (30), lending further support for a role of IL-7 in milk in humans as well. Thus, our observation of a higher proportion of circulating naive $\mathrm{T}$ cells in BF infants is likely to be caused by differences in thymic function in BF and FF infants. Conversely, increased frequencies of naive $\mathrm{T}$ cells in $\mathrm{FF}$ compared with BF infants were reported in the single study that has examined immune cell composition prospectively in relation to early feeding (17). Further, the active phenotype, HLA-DR-CD3+, in the present study varied between 10 and $15 \%$ at all samplings irrespective of feeding mode, which is considerably higher compared with that study, which reported the active phenotype to be $<5 \%$ from 1.5 to 6 months of age (17). Differences in the rates and duration of initial breastfeeding in infants who were later weaned to formula and in environmental antigenic exposures in these two settings (Sweden versus China) might contribute to these inconsistencies.

We also examined the proportion of double-positive CD3 $+\mathrm{CD} 4+\mathrm{CD} 8+\mathrm{T}$ cells. As expected (31), the proportion of this subset of circulating immature $\mathrm{T}$ cells was low, $<2 \%$ in all groups, but it was still slightly higher in the combined formula group during the intervention compared with the BF group. $\mathrm{BF}$ infants also had a higher proportion of $\alpha \beta \mathrm{T}$ cells, whereas the proportions of $\gamma \delta \mathrm{T}$ cells and $\alpha \beta \gamma \delta \mathrm{T}$ cells were higher in FF infants compared with BF infants. The first functional population of circulating $\mathrm{T}$ cells in neonates comprises the $\gamma \delta \mathrm{T}$ cells (32). Most studies in humans have focused on the $\mathrm{V} \gamma 9 / \mathrm{V} 82 \mathrm{~T}$ cells, which form the dominant subset in peripheral blood. $\gamma \delta \mathrm{T}$ cells are activated by exposure to microorganisms, as the predominant receptor on circulating $\gamma \delta \mathrm{T}$ cells recognizes a microbial phospholipid antigen on microorganisms including bacteria and also parasites (33). In the absence of the multitude of immunoprotective factors in human milk (2,3), the increased proportions of $\gamma \delta \mathrm{T}$ cells in FF infants might be a consequence of a triggered immune response (34) to environmental antigens in FF infants. This could be of particular importance in early infancy when there is increased susceptibility to infections, as it appears as if $\gamma \delta \mathrm{T}$ cells can form distinct Th- 1 , Th-2, Th-17, $\mathrm{T}_{\mathrm{FH}}$, and $\mathrm{T}$ regulatory-like effector functions (33) with potential to influence both humoral and cell-mediated responses.

In summary, herein we demonstrate that the addition of OPN to formula at a concentration close to that of human milk increases the proportions of circulating $\mathrm{T}$ cells, whereas a 

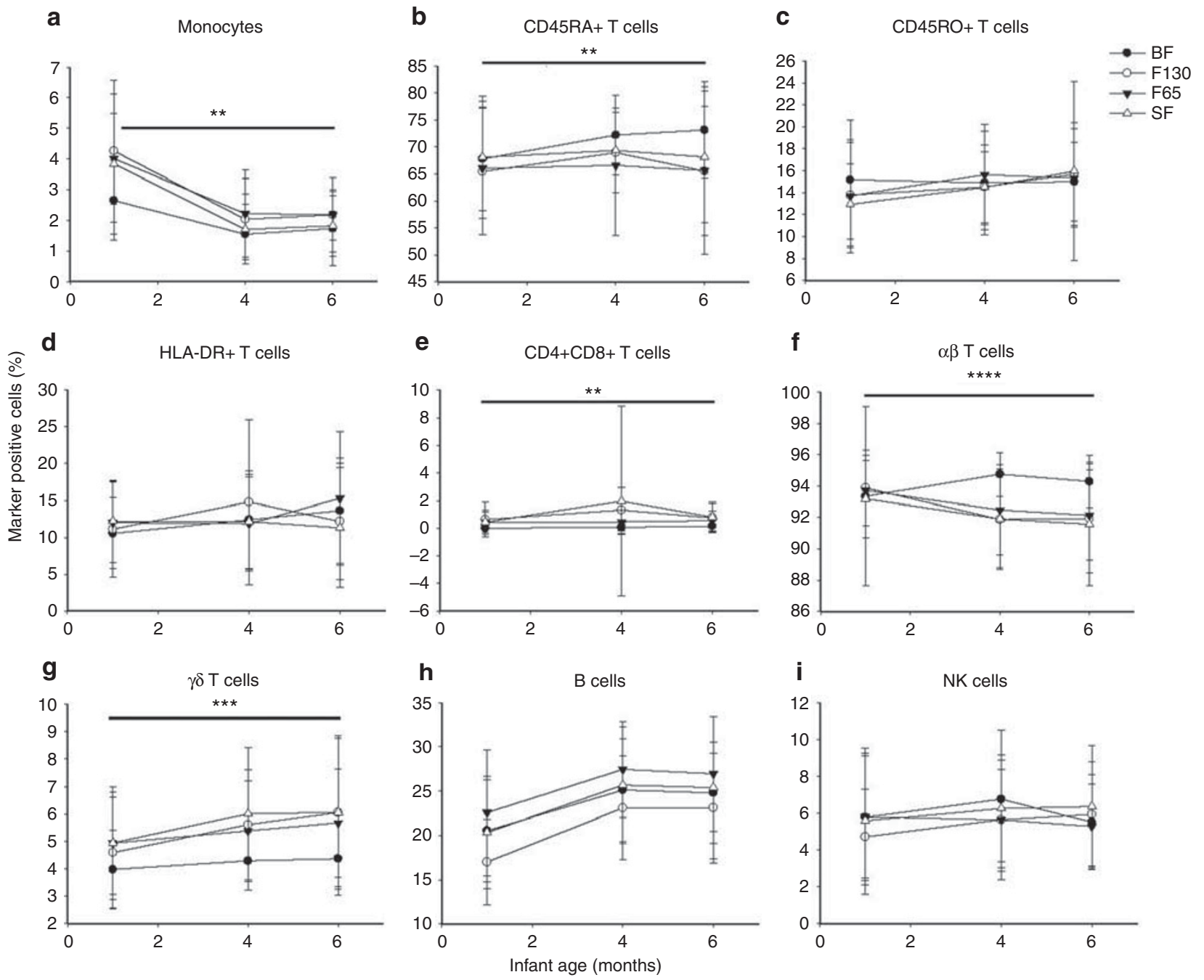

Figure 3. Mean proportions of immune cells as estimated by immunoflow cytometry in PBMCs of infants in the BF reference group and the three formula groups. (a) Monocytes (CD45+CD14+) - BF infants had a lower proportion of monocytes compared with FF infants; the proportion was augmented in the experimental formula groups (F65 and F130). (b) Naive T cells (CD45RA+CD3+) - the proportion of naive T cells was higher in BF infants compared with FF infants. (c) Memory and/or activated T cells (CD45R0+CD3+). (d) HLA-DR T cells (HLA-DR+CD3+ cells). (e) Double-positive T cells $(C D 3+C D 4+C D 8+)$. (f) $\alpha \beta$ T cells (TCR- $\alpha \beta+)$ - the proportion of $a \beta$ T cells was lower in FF infants compared with BF infants. (g) $\gamma \delta T$ cells (TCR$\gamma \delta+$ )-the proportion of TCR- $\gamma \delta+$ cells was higher in FF infants compared with BF infants. (h) B cells (CD19+) and (i) NK cells (CD16+CD56+). Statistically significant differences between the BF group and the combined formula group are indicated; ${ }^{*} P<0.05 ;{ }^{* *} P<0.01$; ${ }^{* * *} P<0.001$; ****P<0.0001. Bars represent mean (SD).

formula with an OPN concentration of 50 or $100 \%$ of that of human milk increases the proportions of monocytes in the first half of infancy with possible implications for the development of the infantile immune system. This calls for further studies investigating the potential role of OPN on $\mathrm{T}$-cell and monocyte function in FF infants. Whether our observations translate into a clinical benefit also remains to be determined; however, significantly fewer days with fever was found in infants fed the F130 formula as compared with the standard formula during the intervention, as reported separately (18). Irrespective of the addition of OPN to infant formula, several differences between $\mathrm{BF}$ and $\mathrm{FF}$ infants were also evident. Breastfeeding was associated with a slightly higher proportion of circulating naive $\mathrm{T}$ cells, which is likely to be due to higher thymic output, whereas FF infants had higher proportions of $\gamma \delta \mathrm{T}$ cells, which may be a consequence of increased antigenic exposure in FF infants. Collectively, this underscores that early infant feeding shapes immune cell ontogeny in early life, with possible short- and long-term health consequences.

\section{METHODS}

\section{Subjects}

From 2009 to 2013, $240 \mathrm{FF}$ infants (120 boys and 120 girls) and a BF reference group with 80 infants ( 40 boys and 40 girls) were recruited in the area of Fudan University, Shanghai, through local advertising, telephone, or posters at the hospital (18). Inclusion criteria were less than one month of age, gestational age of 37-42 weeks at birth, birth weight $>2500 \mathrm{~g}$, absence of chronic illness, and exclusive formulafeeding or, for the BF group, exclusive breastfeeding at inclusion and the mother's intention to exclusively breastfeed until 6 months. Study formulas were delivered to the parents when the infants were about 
three weeks old. Recommendations of feeding only small amounts (taste portions) of complementary foods between four and 6 months of age were given to parents/caregivers. Primary outcomes were growth, health, and immune function assessed at one, four, and 6 months of age. We calculated that with about 70 subjects per group in 4 groups we would be able to detect differences of about $0.56 \mathrm{SD}$ between group means with $80 \%$ power. Another 10 subjects per group were added to allow for dropouts (12\%). Baseline demographic and clinical characteristics for each group have been published previously (18).

\section{Ethics}

The study was approved by the Institutional Review Boards at University of California, Davis and Fudan University, Shanghai. Complete oral and written information about the study was given to the parents/caregivers, and written consent was obtained from parents/caregivers of all infants before inclusion. The study was registered as NCT00970398 under ClinicalTrials.gov at the US NIH.

\section{Randomization}

A customized program was written in SAS for Windows by the biostatistician (J.M.P.) to stratify the FF subjects by sex and randomly assign them to the three formula groups in randomly ordered blocks of three, six, or nine subjects. Subjects were recruited by the study pediatrician (Y-MP) and the research nurses assigned the subjects according to the printed block design. The intervention was blinded to both parents/caregivers and staff until all infants had completed the study. Powdered formula was distributed to families together with preparation instructions in identical cans marked with a code, prepared at the manufacturing site before being delivered to the study site (18).

\section{Study Formulas}

The standard formula (SF) was cow's milk-based (BiosTime Premium, marketed by BiosTime, Guangzhou, China) and the two experimental formulas were modified from this formula. The nutrient composition of this formula is shown in Table 1. The two experimental formulas were fortified with bovine osteopontin (Lacprodan OPN-10, Arla Foods Ingredients, Viby, Denmark) at either $65 \mathrm{mg} / \mathrm{l}$ (F65) or $130 \mathrm{mg} / \mathrm{l}$ (F130), respectively, which corresponds to about 50 and $100 \%$ of the human milk OPN concentration. All three formulas were manufactured by Laiterie de Montaigu (Montaigu, France) and analyzed to contain target concentrations for all ingredients. Volumes of each formula meal and volume or weight of all complementary foods (all groups) were measured and recorded by the parents/caregivers using their own personal household measures and kitchen utilities. Dietary intake, anthropometrics, and morbidity data were recorded but are reported separately (18).

\section{Blood Samples and Flow Cytometry Analyses}

Blood samples were taken by venipuncture at one, four, and six months of age. Concentrations of white blood cells, lymphocytes, monocytes, neutrophilic, eosinophilic, and basophilic granulocytes were assessed by FACS analysis at Children's Hospital Fudan University. Within $2-4 \mathrm{~h}$ at room temperature, monocytes were enriched by density centrifugation of blood taken in heparinized tubes on Ficoll-Paque (Pharmacia, Shanghai, China). The number of isolated cells was counted and stained with fluorochrome-labeled direct conjugated mouse monoclonal antibodies: anti-CD3 clone SK7, anti-CD19 clone 4G7, anti-CD4 clone SK3, anti-CD8 clone SK1, anti-CD16+anti-CD56 clones B73.1+MY31, anti- $\alpha / \beta$ TCR clone WT31, anti- $\gamma / \delta$ TCR clone $11 \mathrm{~F} 2$, anti-HLA-DR clone L243, antiCD45R0 clone LUCHL-1, anti-CD45RA clone L48, anti-CD14 clone $\mathrm{MøP} 9$, and anti-CD45 clone 2D1 (Becton-Dickinson, Mountain View, CA, USA). Approximately 15,000 cells were analyzed by four-color immunofluorescence FACS scan using the CELLQUEST software program (BD Biosciences, San Jose, CA, USA). The instrument was calibrated with Calibrate beads (BD Biosciences).
Lymphocytes were distinguished by dual scatter plots and manually gated to include only lymphocytes into the analysis. Results of the proportion of lymphocytes are given as percentage of CD45+ cells. The absolute number was calculated by multiplying the percentage of each subset with the total number of lymphocytes and monocytes in the sample.

\section{Statistical Analyses}

Data were entered using Microsoft Access and Microsoft Excel 2010 (Microsoft, Redmond, WA, USA) and analyzed with SAS for Windows Release 9.3 (Cary, NC, USA). Descriptive statistics were calculated for all variables. Each continuous variable was compared among groups at each time point with ANCOVA, using the normalized rank of the variable as the outcome and controlling for birth weight and sex of the child; binary variables were compared with logistic regression and the same covariates. Pairwise comparisons were carried out with the Tukey-Kramer adjustment. For continuous variables that were measured at multiple time points, mixed models (repeated measures) analysis was conducted; for these models, because there was no true baseline value both the group effect (testing whether the mean value across all time points differed among groups) and the group by time interaction (testing whether the difference among groups varied by time) were of interest. For all of these procedures, tests were done to compare all four groups with each other, or the three formula groups with each other, or the BF group with the combined formula-fed groups. For effect size measures, Cohen's $\mathrm{d}$ was calculated by dividing the difference between means by the pooled standard deviation. Statistical significance was set at $P<0.05$.

\section{ACKNOWLEDGMENTS}

Frida Karlsson Videhult is acknowledged for assistance with the figures. Role of the sponsor: the sponsor did not participate in the conduct of the study; collection, management, analysis, or interpretation of the data; or preparation of the manuscript. A.S.K. was involved in the design of the study, as well as review and approval of the manuscript.

\section{STATEMENT OF FINANCIAL SUPPORT}

The study was supported by Arla Food Ingredients.

Disclosure: C.E.W., S.M.D., Y.P., and B.L. have received research support from Arla Food Ingredients. A.S.K. is employed by Arla Food Ingredients.

\section{REFERENCES}

1. Nutrition ECo, Agostoni C, Braegger C, et al. Breast-feeding: a commentary by the ESPGHAN Committee on Nutrition. J Pediatr Gastroenterol Nutr 2009;49:112-25.

2. Lönnerdal B. Infant formula and infant nutrition: bioactive proteins of human milk and implications for composition of infant formulas. Am J Clin Nutr 2014;99:712S-7S.

3. Lönnerdal B. Human milk proteins: key components for the biological activity of human milk. Adv Exp Med Biol 2004;554:11-25.

4. West CE, Videky DJ, Prescott SL. Role of diet in the development of immune tolerance in the context of allergic disease. Curr Opin Pediatr 2010;22:635-41.

5. Rautava S, Luoto R, Salminen S, Isolauri E. Microbial contact during pregnancy, intestinal colonization and human disease. Nat Rev Gastroenterol Hepatol 2012;9:565-76.

6. Winkler B, Aulenbach J, Meyer T, et al. Formula-feeding is associated with shift towards Th1 cytokines. Eur J Nutr 2015;54:129-38.

7. Donovan SM, Monaco MH, Drnevich J, et al. Bovine osteopontin modifies the intestinal transcriptome of formula-fed infant rhesus monkeys to be more similar to those that were breastfed. J Nutr 2014;144: 1910-9.

8. Kahles F, Findeisen HM, Bruemmer D. Osteopontin: A novel regulator at the cross roads of inflammation, obesity and diabetes. Mol Metab 2014;3: 384-93. 
9. Lund SA, Giachelli CM, Scatena M. The role of osteopontin in inflammatory processes. J Cell Commun Signal 2009;3:311-22.

10. Shinohara ML, Jansson M, Hwang ES, et al. T-bet-dependent expression of osteopontin contributes to T cell polarization. Proc Natl Acad Sci USA 2005;102:17101-6.

11. Kollmann TR, Levy O, Montgomery RR, Goriely S. Innate immune function by Toll-like receptors: distinct responses in newborns and the elderly. Immunity 2012;37:771-83.

12. Rollo EE, Hempson SJ, Bansal A, et al. The cytokine osteopontin modulates the severity of rotavirus diarrhea. J Virol 2005;79:3509-16.

13. Ashkar S, Weber GF, Panoutsakopoulou V, et al. Eta-1 (osteopontin): an early component of type-1 (cell-mediated) immunity. Science 2000;287: $860-4$.

14. Nau GJ, Liaw L, Chupp GL, et al. Attenuated host resistance against Mycobacterium bovis BCG infection in mice lacking osteopontin. Infect Immun 1999;67:4223-30.

15. Nau GJ, Chupp GL, Emile JF, et al. Osteopontin expression correlates with clinical outcome in patients with mycobacterial infection. Am J Pathol 2000;157:37-42.

16. Pabst HF, Godel J, Grace M, et al. Effect of breast-feeding on immune response to BCG vaccination. Lancet 1989;1:295-7.

17. Andersson Y, Hammarström ML, Lönnerdal B, et al. Formula feeding skews immune cell composition toward adaptive immunity compared to breastfeeding. J Immunol 2009;83:4322-8.

18. Lönnerdal B, Kvistgaard AS, Peerson JM, et al. Growth, nutrition, and cytokine response of breast-fed infants and infants fed formula with added bovine osteopontin. J Pediatr Gastroenterol Nutr 2016;62: 650-7.

19. Bachmann MF, Oxenius A. Interleukin 2: from immunostimulation to immunoregulation and back again. EMBO Rep 2007;8:1142-8.

20. O'Regan AW, Chupp GL, Lowry JA, et al. Osteopontin is associated with $\mathrm{T}$ cells in sarcoid granulomas and has $\mathrm{T}$ cell adhesive and cytokine-like properties in vitro. J Immunol 1999;162:1024-31.

21. Carver JD, Pimentel B, Wiener DA, et al. Infant feeding effects on flow cytometric analysis of blood. J Clin Lab Anal 1991;5:54-6.

22. de Vries E, de Bruin-Versteeg S, Comans-Bitter WM, et al. Longitudinal survey of lymphocyte subpopulations in the first year of life. Pediatr Res 2000;47:528-37.
23. Shearer WT, Rosenblatt HM, Gelman RS, et al. Lymphocyte subsets in healthy children from birth through 18 years of age: the Pediatric AIDS Clinical Trials Group P1009 study. J Allergy Clin Immunol 2003;112: 973-80.

24. Shey MS, Nemes E, Whatney W, et al. Maturation of innate responses to mycobacteria over the first nine months of life. J Immunol 2014;192: 4833-43.

25. Hasselbalch H, Jeppesen DL, Engelmann MD, et al. Decreased thymus size in formula-fed infants compared with breastfed infants. Acta Paediatr 1996;85:1029-32.

26. Hasselbalch H, Engelmann MD, Ersboll AK, et al. Breast-feeding influences thymic size in late infancy. Eur J Pediatr 1999;158:964-7.

27. McCune JM, Loftus R, Schmidt DK, et al. High prevalence of thymic tissue in adults with human immunodeficiency virus-1 infection. J Clin Invest 1998;101:2301-8.

28. van Lent AU, Dontje W, Nagasawa M, et al. IL-7 enhances thymic human T cell development in "human immune system" Rag2-/-IL-2Rgammac-/mice without affecting peripheral $\mathrm{T}$ cell homeostasis. J Immunol 2009;183:7645-55.

29. Ngom PT, Collinson AC, Pido-Lopez J, et al. Improved thymic function in exclusively breastfed infants is associated with higher interleukin 7 concentrations in their mothers' breast milk. Am J Clin Nutr 2004;80: $722-8$.

30. Aspinall R, Prentice AM, Ngom PT. Interleukin 7 from maternal milk crosses the intestinal barrier and modulates $\mathrm{T}$-cell development in offspring. PLoS ONE 2011;6:e20812.

31. Blue ML, Daley JF, Levine H, Schlossman SF. Coexpression of T4 and T8 on peripheral blood $\mathrm{T}$ cells demonstrated by two-color fluorescence flow cytometry. J Immunol 1985;134:2281-6.

32. Gibbons DL, Haque SF, Silberzahn T, et al. Neonates harbour highly active gammadelta $\mathrm{T}$ cells with selective impairments in preterm infants. Eur J Immunol 2009;39:1794-806.

33. Caccamo N, Todaro M, Sireci G, et al. Mechanisms underlying lineage commitment and plasticity of human gammadelta $\mathrm{T}$ cells. Cell Mol Immunol 2013;10:30-4.

34. Roden AC, Morice WG, Hanson CA. Immunophenotypic attributes of benign peripheral blood gammadelta $\mathrm{T}$ cells and conditions associated with their increase. Arch Pathol Lab Med 2008;132:1774-80. 